\title{
The roles of miRNAs in human breast cancer and canine mammary tumor
}

\author{
Richard Ming Chuan Yu ${ }^{1}$ and Yoke Kqueen Cheah ${ }^{1,2,3^{*}}$ (i)
}

\begin{abstract}
MicroRNAs have become a hot topic in cancer research nowadays due to their important role not only on cancer development, progression, invasion but also on repression of cancer related genes. With advanced technologies, these microRNAs can easily be detected from biopsy samples and blood for early diagnosis, prognosis and treatment. Due to increasing demand of research in exploring expression profile of microRNAs with respect to different subtypes of breast cancer, this review aimed to provide an update on microRNA database available resources, canine breast cancer models, the role of microRNA as oncomir or oncosupressor, detection of microRNAs and potential of miRNAs for breast cancer treatment.
\end{abstract}

Keywords: Breast cancer, MicroRNAs, Oncomir, Oncosupressor, Targets of miRNA, Breast cancer model, miRNA profiles, miRNA database

\section{Background}

MicroRNA (miRNA) was discovered in 1993 in Caenorhabditis elegans [1]. These non-coding short RNAs known as miRNAs from eukaryotes such as in plants and animals are highly important for regulation of gene expression at post-transcriptional level. Further, miRNAs are also responsible for cellular growth, differentiation, proliferation and apoptosis [2]. Predominant expression of a particular miRNA varies according to the type of animal tissue. Changes of normal miRNA expression levels indicate abnormal or disease conditions of tissues or body systems. Researchers were convinced to focus more about miRNAs after they found the first miRNA lin-4 of C. elegans that inhibited the expression of lin-14 gene [1]. Since then researchers introduced more and more worthy new findings related to several miRNAs and their roles in different diseases and cancers including breast cancers that potentially led clinicians to be able use circulating miRNAs as affordable, non-invasive detectable biomarkers for earlier diagnosis, prognosis and treatments of breast cancers.

\footnotetext{
* Correspondence: ykcheah@upm.edu.my

${ }^{1}$ Department of Biomedical Science, Faculty of Medicine and Health

Sciences, University Putra Malaysia, 43400 Serdang, Selangor Darul Ehsan,

Malaysia

${ }^{2}$ Centre for Diagnostic Nuclear Imaging, University Putra Malaysia, 43400

Serdang, Selangor Darul Ehsan, Malaysia

Full list of author information is available at the end of the article
}

\section{Processing of microRNAs in the body}

MicroRNAs (miRNAs, miRs) are 20 to 23 nucleotide RNAs and modulate gene expressions [3]. MicroRNAs processing in our body can be observed two parts as they started working in the nucleus of cells and continue its further processing in the cytoplasm [4]. The process in the nucleus initiates with the transcription of noncoding miRNAs genes with their own promoters. These primary transcriptions are carried out mainly by RNA polymerase II and the products are known as primiRNAs [5]. The stem-loop structure of pri-miRNAs are further processed by enzyme Drosha and dsRNAbinding protein DGCR8 [6-8]. The cleavage of primiRNAs produces 70 nucleotide long pre-miRNAs [9]. These pre-miRNAs are then exported into the cytoplasm by exportin 5 for further processing [10]. In the cytoplasm, pre-miRNAs are cleaved by RNase III enzyme, Dicer 1 that works together with transactivation responsive RNA binding protein 2 (TRBP) and AGO2 to generate double stranded miRNA-miRNA* duplex [11-13]. From the two miRNA strands, the guide strands or mature miRNA strands are incorporated into RISC (RNAinduced silencing complex) in order to bind its complementary target mRNA sequences of the target genes to block translation process. The passenger strands miRNA* are degraded $[14,15]$. However, it was also reported that some miRNAs; e.g. miRNA 145 are processed without Dicer, alternative precursors of miRNAs called mirtrons are processed without Drosha. Some 
miRNAs are processed along the pathway of tRNA [1619]. Up to now, scientists have found miRNAs 2619 in human [20].

The process of miRNA begins in the nucleus of cells and continue in the cytoplasm of cells. Primary miRNA (pri-miRNA) transcription is done mainly by RNA polymerase II. Drosha and DGCR8 trim $100 \mathrm{nt}$ to $120 \mathrm{nt}$ long pri-miRNA to become a shorter 70 nt pre-miRNA and it is exported into cytoplasm by exportin 5 for further processes. Pre-miRNA in the cytoplasm is cleaved by RNase III, Dicer I and Argonaute 2 (AGO2) to become a $20 \mathrm{nt}$ to $30 \mathrm{nt}$ long duplex miRNA consists of a guide strand and a passenger strand. From the duplex miRNA, the strand, $3^{\prime}$ to $5^{\prime}$ miRNA known as passenger miRNA is degraded due to PIWI domain in RISC. The guide strand $5^{\prime}$ to $3^{\prime}$ miRNA which is engaged with PAZ domain in RISC binds to its target mRNAs and causes translational repression and cleavage of target mRNAs (Fig. 1).

\section{Mechanisms of miRNA action}

Single stranded mature microRNAs bind 3' UTR (untranslated region) of target mRNAs as a post transcriptional regulation of gene expressions that inhibits translation of target mRNAs for proteins or cleavage of the target mRNAs by Argonaut ribonuclease of RISC (RNA-induced silencing complex) [21]. MicroRNA does not need to bind its whole length of complete nucleotide sequences to target mRNA but miRNA can perform gene expression regulation as long as minimum 2 to 8 base pairs are perfectly complementary between 3 ' region of target mRNA and $5^{\prime}$ region of miRNA (miRNA seed sequence) in human and animals [22]. In human and animals, seed region of miRNA is usually $100 \%$ complementary to target mRNA but the rest sequences of miRNA may include mismatches to target mRNA that causes distended appearance due to limited base paring [23]. Two silencing mechanisms; slicer dependent that shows targeted mRNA cleavage irreversibly by Ago 2 and slicer independent way that causes reversible translation repressions [24].

One miRNA can regulate concurrently the expression of many genes and thus it is involved in multiple cellular signaling pathway [3]. Furthermore, in recent studies, miRNAs was found to be involved in increasing the translation of a target mRNA directly and they were also be able to indirectly increase target mRNA levels by interacting repressor proteins which deter the translation of target mRNA [25]. MicroRNAs (miRNAs), either be as oncogenes or tumor suppressor genes play crucial role in cell differentiation, development, apoptosis and cell cycle. MicroRNAs are known to be expressed differentially in cancers but they show unique expression signature for a specific tumor type. It was reported that
miRNAs influence on metastasis and resistance to different types of therapies [26]. Furthermore, researchers have found pro and anti-metastatic miRNA [27].

\section{MicroRNAs for diagnosis, prognosis and therapeutics}

Detecting circulating miRNA has also become a promising diagnostic tool. MicroRNA profiles are useful not only for diagnosis and prognosis but also for treatments in terms of choosing effective chemotherapeutic drug in cancers. This is why nowadays detecting miRNA profiles from breast cancer become more and more common in research field and also this happens to clinicians. In a study with zebra fish, researcher found that certain miRNAs dominantly expressed in specific tissues and they became noticeably reduced expression levels when the tissues are in tumors state [28]. Commonly used breast cancers diagnostic methods such as mammography, ultrasound, X-rays and MRI still exist limitations of its use in patients and also required skills to read the images for proper interpretations. Although detecting circulating miRNA is not a substitute for other conventional diagnostic methods, it simply becomes essential for most cancer diseases due to its less invasive than tissue biopsy which has to be done for genetic tests such as specific mRNA expression. It has been reported several miRNAs have been proved to be deregulated. Up-regulated miRNAs that may be used for diagnosis, prognosis and therapeutic are miR21, miR155, miR221/ 222, miR9, miR10b, miR29a, miR96, miR146a, miR181, miR373, miR375, miR520c and miR589 [29, 30]. Some of these up-regulated miRNAs in tumors play major role in controlling and working together with multiple targets genes and led them to be invasive types. Downregulated miRNAs are those with tumor suppressor properties. Recent study reported that let7c is possible biomarker which can discriminate breast cancer samples grade 1 to grade 3 [3]. Other consistently downregulated miRNAs are miR30a, miR31, miR34, miR92a, miR93, miR125, miR126, miR146a, miR195, miR200, miR205, miR206, miR503 and let7 family.

In breast cancer stem cells, miR15/16, miR103/107, miR128b, miR145, miR200 and miR335 are found to be down-regulated. With great efforts, now total 130 over miRNAs signatures were identified to distinguish between normal and tumor breast tissue accurately [31]. MicroRNAs in breast cancers can be classified into Oncomirs, Oncosupressors and MetastamiRs [32]. Oncomirs disturb expression of oncosupressors and cells apoptosis. In human breast cancer, oncomirs miRNA21 and miRNA155 are outstandingly upregulated and oncosupressors miRNA10b, miRNA125b and miRNA145 are noticeably downregulated. In triple negative breast cancers, miRNA21, miRNA 210 and miRNA 221 were 


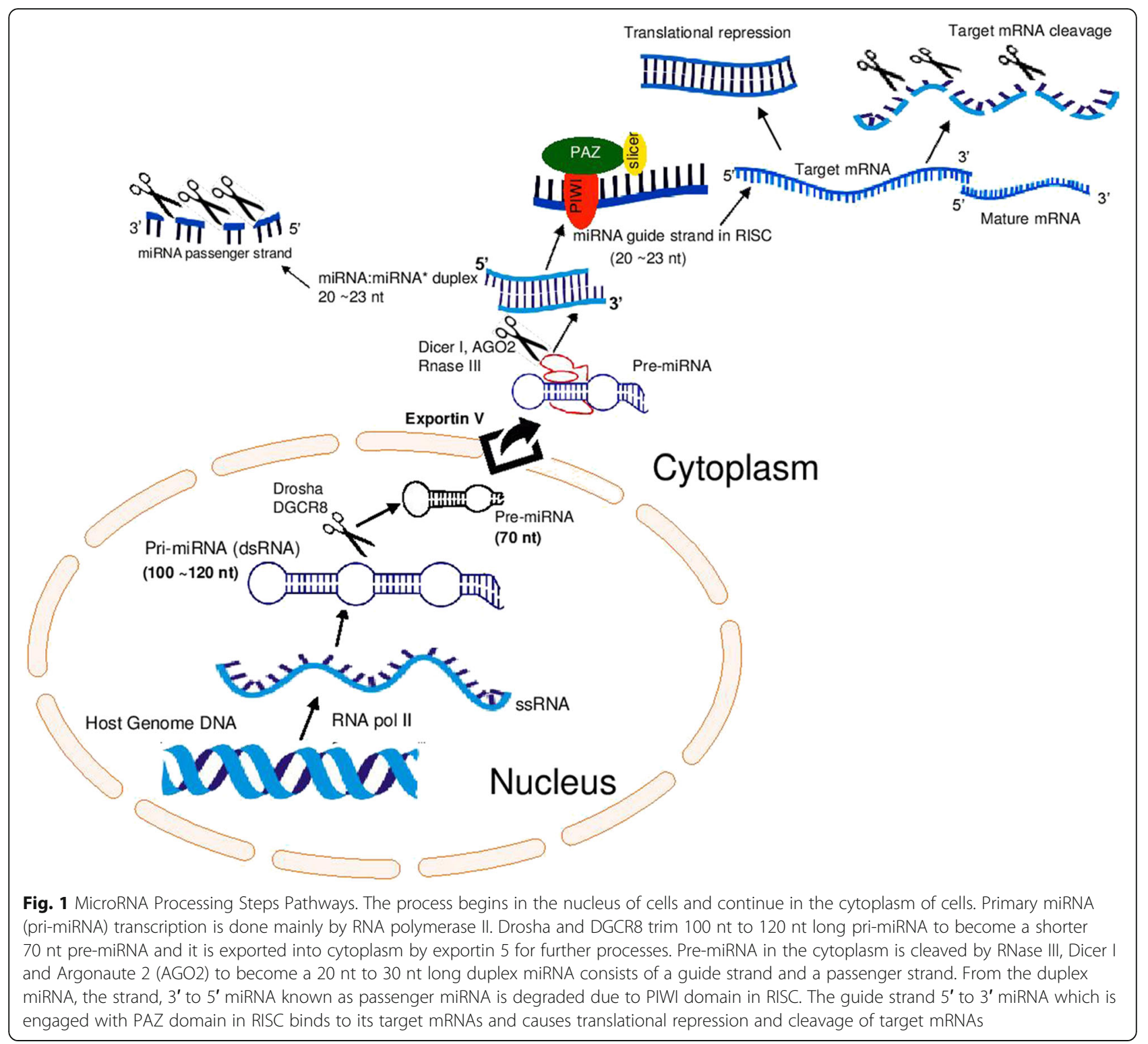

found to be upregulated. Anti-miRNAs to those upregulated oncomirs not only inhibited cancer cells growth but also increased apoptosis and decreased cell proliferation. Oncosupressors such as miR204, miR34a,b,c and let-7 family of microRNAs are found to be downregulated in breast cancers. In one study, let- 7 mimics showed effectiveness in the treatment of lungs cancer in mouse model when it was administered I.V. in the form of neutral lipid emulsion. MetastamiRs are recently recognized and introduced to those small non-coding miRNAs that regulates migration and invasion of cancer cells $[33,34]$. These metastamiRs plays as major role in epithelial-mesenchymal transition (EMT), apoptosis and angiogenesis. It was reported that miRNAs $10 \mathrm{~b}$ expression is $50 \%$ higher in invasive type of cancer cell line MDA-MB-231 compare to non-invasive type MCF-7 cancer cell line. Although miRNA10b was found to be down-regulated in non-invasive breast cancers, its change to up-regulation indicates the initiation of breast cancer metastasis. A study shown that applying antisense miR10b inhibitor oligonucleotides reduces invasiveness of transfected cells. It is required to restore the level of miRNAs to normal state and this can be achieved by dosing miRNA mimics or inhibitors, introducing miRNA genes with a DNA vector, application of small molecules to reverse epigenetic silencing of miRNAs [28]. MicroRNAs mimics are short double stranded RNA guide strand and passenger stand oligonucleotides. They may be fully or partially complementary each other. The list of down-regulated miRNAs has been published and each of them are indeed potential cancer therapeutic miRNAs. It was also known that one therapeutic miRNA is 
able to target multiple mRNAs. In vivo study further provided an evidence that silencing of overexpression miR10b with systemic antagomirs suppressed breast cancer metastasis in mice [35]. Another study reported that miR373 was up-regulated in patients with breast cancer metastasized to lymph nodes. Like other circulating miRNAs, metastamiRs also become a popular molecular marker for breast cancers. Circulating miRNAs biomarkers offered affordable and non-invasive method of early detection, differentiating subtypes of breast cancers which allows clinicians to predict prognosis and further adjust their treatment regime as required. Table 1 miRNAs expression level, their targets, functional roles and potentials as biomarker in human breast cancer (female).

\section{Methods to detect miRNAs in breast cancer}

Micro-RNAs are quite a smaller size and impossible to detect them with the methods used for mRNA. However, since miRNAs roles become important and also found to be significant biomarkers of diseases including cancers, scientists invested enormous efforts in order to detect them. Nowadays, about 30 methods in total are available to detect miRNA expression profiles. MicroRNA expression profiles in certain diseases, give critical useful information about diagnosis and prognosis. Among these miRNA detection methods, commonly applied to detect miRNA are Northern blotting, Microarray, Bead based flow cytometry, qRT-PCR, In sithu hybridization and Next-Generation Sequencing [36]. Among them, the most standardized method for miRNA analysis is Northern blotting although it is lowthroughput [37].

Being a high throughput, miRNA microarrays can be applied several miRNAs in a large number of samples. However, miRNA microarrays is low specificity and low sensitivity due to their extremely short sequence which allows only very little room to fine-tune the hybridization conditions of miRNAs [38]. Another high throughput, high specificity and high sensitivity method for assessment of miRNA is qRT-PCR and this method can also be used for validation of data obtained from other detection platforms [39]. Bead-based flow cytometry and in situ hybridization detection methods are limited to known miRNA structures. However, the latest detection method which gives high throughput, high specificity and high sensitivity is Next-generation sequencing and this method allows to discovery of new miRNA as well as to confirm known miRNA [40].

In breast cancers, miR10b, miR125b and miR145 are consistently found to be down-regulated and miR21, miR155 are consistently up-regulated [41, 42]. It was also reported that miRNAs can still be detected from formalin fixed and paraffin embedded tissue samples, collected blood and serum too. MicroRNAs are stable due to their smaller size that showed tolerance to ribonuclease degradation.

\section{Breast cancer model}

All previous studies have identified that canine mammary tumor models are great to study the molecular pathogenesis of human breast cancer due to several similarities such as epidemiological factors and histopathological aspects [43-46]. In addition, it is very common that dogs and humans are exposed to equivalent carcinogens as frequently they are living in the same environment. Risk factors and molecular changes in affected mammary tissues for both species are comparable. Most of canine mammary tumors and human breast cancers are also originated from epithelial tissues [47]. In fact, different types of human cancers can be found in dogs too. For example, lymphoma, breast cancer, bone cancer and etc. Furthermore, studies have pointed out specifically that the age of onset and peak incidence among humans and dogs are about the same if their age is calculated proportionately [43]. Spontaneous mammary tumors incidence rate in female dogs is about $25 \%$ ( 1 in 4 ) while breast cancer incidence rate in women is about $12 \%$ ( 1 in 8 ). Readily availability of spontaneous canine mammary tumors is one of the factors that makes them a good model to study breast cancer as an alternative to experimental animal models. In particular, similarities including expression of hormone receptors, tumor growth markers, epidermal growth factor receptor, and P53 mutations also strengthen the canine mammary tumor model. Moreover, overexpression of cyclooxygenase-2 (COX-2), a molecule directly involved with mammary carcinogenesis, and matrix metalloproteinase-2 (MMP-2) which plays a role in invasion and metastasis process was detected in both species too $[48,49]$.

It is noted that miRNA genes between human and canine are highly conserved. A study had been reported that miR15a, miR16, miR17-5p, miR21, miR29b, miR125b, miR155, miR181b and let7 family expression patterns were the same between human breast cancer and canine mammary tumors except miR145 which does not showed changes in canine [50,51]. As in human breast cancer, miR15a and miR16 expressions were also reduced in canine mammary carcinomas. Furthermore, miR15a and miR16 were shown to be involved in differentiation of malignancy [51]. Computationally analysis results showed that 300 miRNAs amongst canine miRNAs were found to have the same sequences as of those found in human [52]. Also, it has been described that miRNA210 which is regulated by hypoxia, was significantly upregulated in continuous manner throughout canine mammary tumor progression [53]. In human breast 
Table 1 miRNAs expression level, their targets, functional roles and potentials as biomarker in human breast cancer (female)

\begin{tabular}{|c|c|c|c|c|c|}
\hline $\begin{array}{l}\text { miRNA } \\
\text { Human } \\
\text { (Female) }\end{array}$ & Sample Source & $\begin{array}{l}\text { Expression } \\
\text { Level }\end{array}$ & Target & $\begin{array}{l}\text { Functional Role, Biomarker as Diagnostic, } \\
\text { Prognostic, Therapeutic }\end{array}$ & Authors, year of publication \\
\hline $\begin{array}{l}\text { Let } 7 \\
\text { family }\end{array}$ & $\begin{array}{l}\text { Cell line, } \\
\text { Tissue, Blood, } \\
\text { Serum }\end{array}$ & Down & $\begin{array}{l}\text { RAS, HMGA2, H-RAS, } \\
\text { KRAS, MYC, CCND2, } \\
\text { PBX3, LIN28, PEBP1, BMI- } \\
1\end{array}$ & $\begin{array}{l}\text { Proliferation, differentiation, self-renewal, } \\
\text { EMT, Tamoxifen response, diagnostic and } \\
\text { prognostic biomarker }\end{array}$ & $\begin{array}{l}\text { Kurozumi et al. } 2016 \text { [57], Bertoli et al. } \\
2015 \text { [3] } \\
\text { Schooneveld et al. } 2015 \text { [36], Kaboli } \\
\text { et al. } 2015 \text { [58], Takahashi et al. } 2015 \\
\text { [59], Zhang et al. } 2014 \text { [60], } \\
\text { Schwarzenbacher et al. } 2013 \text { [61], Fu } \\
\text { et al. } 2011 \text { [62], O' Day et al. } 2010 \text { [2] }\end{array}$ \\
\hline
\end{tabular}

$7 \quad$ Tissue $\quad$ Down FAK, IGFR, EGFR, REGY

ER+ tissue, Up

Cell line

10b Cell line, Up

Tissue TIAM1, E-CAD, RHOC

Plasma, Tissue, Down

Cell line,

Serum

17-5p Tissue

Down

E2F1, CCND1, AIB1

\begin{tabular}{|c|c|c|c|}
\hline $17 / 92$ & Cell line & Up & $\begin{array}{l}\text { E2Fs, ERa, C-MYC, AlB1, } \\
\text { CYCLIN D1, MEKK2 }\end{array}$ \\
\hline 21 & $\begin{array}{l}\text { Plasma/Tissue/ } \\
\text { Cell line, } \\
\text { Serum, Blood }\end{array}$ & Up & $\begin{array}{l}\text { PTEN, BCL-2, TPM1, } \\
\text { TIMP3, HER, PDCD4, } \\
\text { MASPIN, CDC25, PTEN, } \\
\text { BCL2, RHOB, MMPs, } \\
\text { HIF1A }\end{array}$ \\
\hline 22 & Cell line & Down & $\begin{array}{l}\text { CDK6. SIRT1, SP1, TET1- } \\
\text { 3, TIP60 }\end{array}$ \\
\hline
\end{tabular}

Tissue

Up

TNBC, Cell line Up and tissue

$\begin{array}{llll}\text { 29b } & \text { Tissue } & \text { Down } & \begin{array}{l}\text { ITGB1, MMP2, TIAM1, } \\ \text { VEGFA, ANGPTL4, LOX }\end{array} \\ 30 & \begin{array}{l}\text { TNBC, Cell line } \\ \text { and BCSCs }\end{array} & \text { Down } & \text { UBC9, ITGB3, AVEN } \\ \text { 30c } & \text { Tissue } & \text { Down } & \text { TWF1, IL-11, VIM }\end{array}$

WIP1, BCL2, E2F, CDK6, CCND1 MYT-1, ZBTB10

CYP1B1, ARFGEF1, FOXO1, PPARY, ST14, $\mathrm{NISCH}$
Regulating gene expression, cell growth Bertoli et al., 2015 [3], Kaboli et al. 2015 and survival control, Tamoxifen response, [58]

Docetaxel and Cisplatin sensitive,

diagnostic biomarker

Regulate CDKs and control cell cycle progression G1 to S, cellular adhesion and metastasis, diagnostic and prognostic biomarker

Promote cell migration, invasion, metastasis, EMT, stemness of BCSC, Tamoxifen resistance, diagnostic biomarker

Regulate cell proliferation and death, diagnostic and prognostic, Doxorubicin and Docetaxel response

Regulate CDKs and control cell cycle progression G1 to $\mathrm{S}$, cancer cell proliferation, diagnostic biomarker

Control cell cycle, proliferation, tumorigenesis, proapoptosis, metastasis

Promote cell migration, invasion, metastasis, EMT, diagnostic and prognostic biomarkers, resistant to Cisplatin, Doxorubicin, Topotecan

Regulator of cellular senescence, inhibit tumor growth and metastasis, regulate EMT genes by repressing TIP60, prognostic biomarker (TIP60/miR-22)

FOXO1, ZBTB10/RINZF, Cell cycle progression G2 to M check point regulation, tumor development, invasion and metastasis, diagnostic and prognostic biomarker

Regulate cell cycle progression, proliferation, metastasis, angiogenesis, drug resistance, generation of breast cancer stem cells (BCSCs), prognostic biomarker

Inhibit proliferation, angiogenesis and metastasis. Diagnostic biomarker

Regulate self-renewal and antiapoptotic, in-vitro mammosphere formation.

Tamoxifen response, suppresses interleukin 11 expression and inhibit resistance to paclitaxel and doxorubicin
Bertoli et al., 2015 [3], Kaboli et al. 2015 [58],

Schooneveld et al. 2015 [36], Zhang et al. 2014 [60]

Bertoli et al. 2015 [3], Schooneveld et al. 2015 [36]

Kaboli et al. 2015 [58], Takahashi et al. 2015 [59],

Shafi et al. 2014 [32], Zhang et al. 2014 [60], Fu et al. 2011 [62], O' Day et al. 2010 [2]

Bertoli et al. 2015 [3], Kaboli et al. 2015 [58],

Zhang et al. 2014 [60], Schwarzenbacher et al. 2013 [61],

Kurozumi et al. 2016 [57], Bertoli et al. 2015 [3],

Fu et al. 2011 [62], O’ Day et al. 2010 [2]

Kaboli et al. 2015 [58], Schooneveld et al. 2015 [36], Xiang et al. 2010 [63], Bonauer et al. 2009 [64]

Kurozumi et al. 2016 [57], Bertoli et al. 2015 [3],

Schoonevel et al. 2015 [36], Kodahl et al.2014 [65], Shafi et al. 2014 [32], Zhang et al. 2014 [60]

Kaboli et al. 2015 [58], Takahashi et al. 2015 [59], Schwarzenbacher et al. 2013 [61]

Kurozumi et al. 2016 [57], Bertoli et al. 2015 [3], Zhang et al. 2014 [60], Tang et al. 2012 [66], Fu et al. 2011 [62], Mertens-Talcott et al. 2007 [67]

Ding et al. 2017 [68], Kurozumi et al. 2016 [57], Bertoli et al. 2015 [3], Zhang et al. 2014 [60], Schoonevel et al. 2015 [36], Fu et al. 2011 [62]

Kurozumi et al. 2016 [57], Bertoli et al. 2015 [3]

Bertoli et al. 2015 [3], Kaboli et al. 2015 [58]

Schwarzenbacher et al. 2013 [61]

Kurozumi et al. 2016 [57], Bertoli et al. 2015 [3],

Takahashi et al. 2015 [59] 
Table 1 miRNAs expression level, their targets, functional roles and potentials as biomarker in human breast cancer (female) (Continued)

\begin{tabular}{llll}
\hline 31 & BC and TNBC Down & RHOA, RDX, ITGA5, & Inhibits several steps of the invasion- \\
cell line & & FZD3, M-RIP, MMP16, & metastasis cascade in breast cancer \\
& WAVE3, PKCepilon &
\end{tabular}

$\begin{array}{lll}\text { 34a, b, c } & \begin{array}{l}\text { BC and TNBC Down } \\ \text { cell line, } \\ \text { Tissue }\end{array} & \\ 125 a-5 p & \begin{array}{l}\text { Cell line, } \\ \text { Tissue }\end{array} & \text { Down } \\ 125 b & \begin{array}{ll}\text { Cell line, } \\ \text { Tissue }\end{array} & \text { Down }\end{array}$

$126 \quad$ BC and TNBC Down cell line, Tissue

Cell line and Down BCSCs

Serum

$$
\text { Down }
$$

Serum/

Plasma/Tissue

Cell line

$B C$ and TNBC Down cell line, Tissue

$B C$ and TNBC Down cell line, Tissue

$\mathrm{BC}$ and TNBC Down cell line, Tissue

Serum, Up

Heterogenous

$\mathrm{BC}$, Tissue

181

Cell line and Up

BCSCs, Serum

181b Tissue, Blood, Up Cell line

Cell line and Up tissue

Tissue

Up
NOTCH4, NOTCH 1, CCND1, AXL, WIP1 C-MYC, FRA1, CDK4, CDK6, SIRT1, E2F3

HDAC4, HDAC5, HER3, HUR

HER2, EST1, E2F3, EPO, EPOR, ENPEP, CK2-a, CCNJ, MEGF9, ERBB2, HUR, BAK

IGFBP2, PITPNC1, MERTK, VEGF, IRS-1, PIK3R2

BMI-1, ABCC5

HER3

EGF, C-MYC, VEGF, NCADHERIN, HIF-2a, MUCIN1, HER3, IRS1, RTKN

BRCA1, NFKB, TRAF6, IRAK1, ROCK1, CXCR4, EGFR

ICAM1, VHRF1, NF-Kb, EGFR

ICAM1, VHRF1, NF-KB, STAT3, EGFR

CXCR4, FOXO3, TRF1, SHIP, TP53INPI, RHOA, SOCS1

ATM

SMAD3, BIM, CDK8

BRCA1, FOXO1

TLN2, CDH2, RAC1, THBS1, ITGA9
Cell Cycle control, invasion and metastasis, EMT, self-renewal and EMT, diagnostic biomarker, radiotherapy sensitive

Inhibit cell proliferation and differentiation, induce apoptosis, Docetaxel sensitive, diagnostic biomarker

Inhibit cell proliferation and differentiation, diagnostic biomarker, FEC chemotherapy resistant, Taxol resistant, Trastuzumab sensitive.

Cell cycle progression from G1/G0 to S, reduces metastasis and angiogenesis, diagnostic biomarker

Regulate cell cycle, inhibit tumor growth and angiogenesis, Doxorubicin sensitive

Inhibit cell invasion and metastasis, diagnostic biomarker

Inhibit cell invasion and metastasis, diagnostic biomarker

Proliferation, antiapoptotic, diagnostic biomarker

Antimetastasis, symmetric and asymmetric division of CSCS

Anti-metastasis

Cell growth, proliferation, metastasis, telomere synthesis, TGF- $\beta$ Signaling, diagnostic and prognostic biomarker, Taxane response

Regulate in vitro mammosphere formation, anti-apoptotic

Promotes cell proliferation, migration and metastasis, associated with the resistance to Doxorubicin, diagnostic biomarker

Proliferation, antiapoptotic, Tamoxifen response

Antimetastastic, inhibit cell migration, enhance chemosensitivity, associate with trastuzumab (Herceptin) response, diagnostic biomarker.
Kurozumi et al. 2016 [57], Bertoli et al.

2015 [3],

Kaboli et al. 2015 [58], Schooneveld

et al. 2015 [36],

Zhang et al. 2014 [60], Fu et al. 2011

[62], O’ Day et al. 2010 [2]

Bertoli et al. 2015 [3], Zhang et al. 2014

[60],

Schwarzenbacher et al. 2013 [61]

Kaboli et al. 2015 [58], Fu et al. 2011 [62]

O' Day et al. 2010 [2]

Hsieh et al. 2015 [69], Bertoli et al. 2015

[3], Kaboli et al. 2015 [58], Zhang et al.

2014 [60]

Kurozumi et al. 2016 [57], Bertoli et al., 2015 [3],

Schooneveld et al. 2015 [36], Zhang

et al. 2014 [60], Feliciano et al. 2013

[70], Wang et al. 2012 [71]

Kurozumi et al. 2016 [57], Bertoli et al. 2015 [3],

Zhang et al.2014 [60], Fu et al. 2011 [62]

Bertoli et al. 2015 [3], Kaboli et al. 2015

[58],

Schwarzenbacher et al. 2013 [61]

Schooneveld et al. 2015 [36], Kaboli et al. 2015 [58], Kodahl et al. 2014 [65]

Bertoli et al. 2015 [3], Schooneveld et al. 2015 [36], Zhang et al. 2014 [60], Kodahl et al. 2014 [65]

Fu et al. 2011 [62]

Bertoli et al. 2015 [3], Shafi et al. 2014 [32]

Kurozumi et al. 2016 [57], Bertoli et al. 2015 [3], Kaboli et al. 2015 [58]

Kurozumi et al. 2016 [57], Schooneveld et al. 2015 [36], Kaboli et al. 2015 [58]

Bertoli et al. 2015 [3], Schooneveld et al. 2015 [36],

Kodahl et al.2014 [65], Zhang et al. 2014 [60], Kaboli et al. 2015 [58], Fu et al. 2011 [62], O’ Day et al. 2010 [2]

Bertoli et al. 2015 [3], Schwarzenbacher et al. 2013 [61], Kaboli et al. 2015 [58]

Zheng et al. 2015 [72], Kaboli et al. 2015 [58]

Kurozumi et al. 2016 [57], Bertoli et al. 2015 [3]

Kaboli et al. 2015 [58], Zhang et al. 2014 [60]

Bertoli et al., 2015 [3], Kaboli et al. 2015 [58], Calura et al. 2014 [73], Le et al. 2012 [74]

Fang et al. 2016 \& $2013[75,76]$ 
Table 1 miRNAs expression level, their targets, functional roles and potentials as biomarker in human breast cancer (female) (Continued)

\begin{tabular}{|c|c|c|c|c|c|}
\hline & $\begin{array}{l}\text { Tissue, Cell } \\
\text { line }\end{array}$ & & & $\begin{array}{l}\text { Regulate proliferation, invasion and } \\
\text { metastasis, prognostic biomarker }\end{array}$ & \\
\hline 200 & $\begin{array}{l}\text { Tissue and Cell } \\
\text { line }\end{array}$ & Down & $\begin{array}{l}\text { BMI-1, SUZ12, ZEB1, } \\
\text { ZEB2, PLCG1, TGFß2, } \\
\text { FAP-1, SNAI-1, SNAI-2, } \\
\text { CTNNB1 }\end{array}$ & $\begin{array}{l}\text { Reduces tumor growth, anti-metastatic, } \\
\text { stemness and EMT. }\end{array}$ & $\begin{array}{l}\text { Bertoli et al. } 2015 \text { [3], Kaboli et al. } 2015 \\
\text { [58], } \\
\text { Shafi et al. } 2014 \text { [32], Hilmarsdottir et al. } \\
2014 \text { [77], Schwarzenbacher et al. } 2013 \\
\text { [61], O' Day et al. } 2010 \text { [2] }\end{array}$ \\
\hline $200 a$ & $\begin{array}{l}\text { Tissue and Cell } \\
\text { line }\end{array}$ & Down & $\begin{array}{l}\text { SLUG, BMI1, ZEB1, ZEB2, } \\
\text { EPHA2 }\end{array}$ & Reduces tumor growth, anti-metastatic & $\begin{array}{l}\text { Tsouko et al. } 2015 \text { [78], Bertoli et al. } \\
2015 \text { [3], Kaboli et al. } 2015 \text { [58] }\end{array}$ \\
\hline $200 \mathrm{~b}$ & $\begin{array}{l}\text { Tissue and Cell } \\
\text { line }\end{array}$ & Down & $\begin{array}{l}\text { SP1, RAB21, RAB23, } \\
\text { RAB18, RAB3B }\end{array}$ & $\begin{array}{l}\text { Control cell proliferation and apoptosis, } \\
\text { Prognostic biomarker }\end{array}$ & Yao et al. 2015 [79], Ye et al. 2014 [80] \\
\hline $200 c$ & $\begin{array}{l}\text { Tissue and cell } \\
\text { line }\end{array}$ & Down & $\begin{array}{l}\text { BMI1, ZEB1, ZEB2, } \\
\text { FHOD1, PPM1F }\end{array}$ & $\begin{array}{l}\text { Reduces tumor growth, antimetastatic, } \\
\text { inhibit clonogenicity of BCSCs, induce } \\
\text { differentiation, sensitize breast cancer } \\
\text { cells to doxorubicin, therapeutic } \\
\text { biomarker }\end{array}$ & $\begin{array}{l}\text { Bertoli et al. } 2015 \text { [3], Kaboli et al. } 2015 \\
\text { [58], } \\
\text { Zhang et al. } 2014 \text { [60], Jurmeister et al. } \\
2012 \text { [81], Fu et al. } 2011 \text { [62], Shimono } \\
\text { et al. } 2009 \text { [82] }\end{array}$ \\
\hline 204 & $\begin{array}{l}\text { Tissue and cell } \\
\text { line }\end{array}$ & Down & $\begin{array}{l}\text { JAK2, ZEB2, FOXA1, } \\
\text { BDNF, IL-11, PDEF, SIX1, } \\
\text { SAM68 }\end{array}$ & $\begin{array}{l}\text { Inhibit cell growth, invasion and } \\
\text { metastasis, induce cell apoptosis, } \\
\text { suppress BCSCs, diagnostic and } \\
\text { prognostic biomarker, Tamoxifen } \\
\text { response }\end{array}$ & $\begin{array}{l}\text { Shen et al. } 2017 \text { [83], Li et al. } 2016 \text { [84], } \\
\text { Flores-Pérez et al. } 2016 \text { [85], Bertoli et } \\
\text { al. } 2015 \text { [3] }\end{array}$ \\
\hline 205 & $\begin{array}{l}\text { Ductal } B C, B C \\
\text { and TNBC cell } \\
\text { line }\end{array}$ & Down & $\begin{array}{l}\text { HER3, E2F, P53, ZEB-1, } \\
\text { HMGB3, VEGF-A, BRBB3 }\end{array}$ & $\begin{array}{l}\text { Inhibit proliferation, invasion and EMT, } \\
\text { prognostic biomarker }\end{array}$ & $\begin{array}{l}\text { Bertoli et al. } 2015 \text { [3], Schooneveld et } \\
\text { al. } 2015 \text { [36], } \\
\text { Takahashi et al. } 2015 \text { [59], Zhang et } \\
\text { al.2014 [60], Fu et al. } 2011 \text { [62] }\end{array}$ \\
\hline 206 & $\begin{array}{l}\mathrm{BC} \text { and TNBC } \\
\text { cell line }\end{array}$ & Down & $\begin{array}{l}\text { ERa, CCND2, ESR1, } \\
\text { NOTCH3, SRC-1, SRC-3, } \\
\text { GATA-3, ERa, CX43 }\end{array}$ & $\begin{array}{l}\text { Reduces migration, invasion and anti- } \\
\text { metastatic }\end{array}$ & $\begin{array}{l}\text { Bertoli et al. } 2015 \text { [3], Schooneveld et } \\
\text { al. } 2015 \text { [36], } \\
\text { Kaboli et al. } 2015 \text { [58], Shafi et al. } 2014 \\
\text { [32], Zhang et al. } 2014 \text { [60], Fu et al. } \\
2011 \text { [62], O' Day et al. } 2010 \text { [2] }\end{array}$ \\
\hline 210 & $\begin{array}{l}\text { IDC, Cell line, } \\
\text { Tissue, Plasma }\end{array}$ & Up & $\begin{array}{l}\text { E2F3, NPTX1, RAD52, } \\
\text { ACVR1B, MNT, } \\
\text { CASP8AP2, FGFRL1, } \\
\text { HOXA-1, HOXA-9 }\end{array}$ & $\begin{array}{l}\text { Prognostic and diagnostic biomarker, } \\
\text { Herceptin resistance }\end{array}$ & $\begin{array}{l}\text { Bertoli et al. } 2015 \text { [3], Schooneveld et } \\
\text { al. } 2015 \text { [36], Devlin et al. } 2011 \text { [86], } \\
\text { Huang et al. } 2010 \text { [87] }\end{array}$ \\
\hline $216 b$ & $\begin{array}{l}\text { Tissue and cell } \\
\text { line }\end{array}$ & Down & SDCBP, P2X7 & $\begin{array}{l}\text { Suppress breast cancer growth and } \\
\text { metastasis, proapoptoic, therapeutic } \\
\text { biomarker }\end{array}$ & $\begin{array}{l}\text { Jana et al. } 2016 \text { [88], Kaboli et al. } 2015 \\
\text { [58], Zheng et al. } 2014 \text { [60] }\end{array}$ \\
\hline 222 & Serum, Tissue & Up & $\begin{array}{l}\text { ABCG2, MMP1, SOD2, } \\
\text { TIMP3, GNAI3 }\end{array}$ & $\begin{array}{l}\text { Inhibit migration and invasion, enhance } \\
\text { breast cancer cells to cisplatin } \\
\text { responsiveness, diagnostic and } \\
\text { prognostic biomarker }\end{array}$ & $\begin{array}{l}\text { Bertoli et al. } 2015 \text { [3], Schooneveld et } \\
\text { al. } 2015 \text { [36], Zhao et al. } 2015 \text { [89], } \\
\text { Kodahl et al. } 2014 \text { [65] }\end{array}$ \\
\hline $221 / 222$ & Cell line & Up & $\begin{array}{l}\text { ERa, P27kip1, KIT, P57, } \\
\text { PTEN }\end{array}$ & $\begin{array}{l}\text { Tamoxifen resistant luminal type breast } \\
\text { cancer and Fulvestrant resistant }\end{array}$ & $\begin{array}{l}\text { Bertoli et al. } 2015 \text { [3], Takahashi et al. } \\
2015 \text { [59] } \\
\text { Piva et al. } 2013 \text { [30], Fu et al. } 2011 \text { [62] }\end{array}$ \\
\hline 224 & $\begin{array}{l}\text { Tissue and Cell } \\
\text { line }\end{array}$ & Up & $\begin{array}{l}\text { CXCR4, CDC42, RKIP, } \\
\text { FZD5, FZD4, }\end{array}$ & Inhibited cell proliferation and migration & $\begin{array}{l}\text { Liu et al. } 2014 \text { [90], Zhang et al.2014 } \\
\text { [60] }\end{array}$ \\
\hline 335 & $\begin{array}{l}\text { Cell line, } \\
\text { Tissue }\end{array}$ & Down & $\begin{array}{l}\text { SOX4, SPL, BCL-W, SOX4, } \\
\text { TNC, PTPRN2, MERTK, } \\
\text { RSP1, IGF1, ID4, ERa }\end{array}$ & $\begin{array}{l}\text { Suppress metastasis and migration, } \\
\text { proapoptotic, diagnostic and prognostic } \\
\text { biomarkers }\end{array}$ & $\begin{array}{l}\text { Bertoli et al. } 2015 \text { [3], Schooneveld et } \\
\text { al. } 2015 \text { [36] } \\
\text { Kaboli et al. } 2015 \text { [58], Shafi et al. } 2014 \\
\text { [32], Zhang et al. } 2014 \text { [60] }\end{array}$ \\
\hline $339-5 p$ & Tissue & Down & BCL6 & Proapoptotic, Tamoxifen response & $\begin{array}{l}\text { Bertoli et al. } 2015 \text { [3], Kaboli et al. } 2015 \\
\text { [58] }\end{array}$ \\
\hline $342-5 p$ & $\begin{array}{l}\text { Heterogenous } \\
\text { BC, Tissue }\end{array}$ & Down & $\begin{array}{l}\text { EGRF, HER2, AKT, PKC, } \\
\text { ESR1, ERN2, PELP1, SRC }\end{array}$ & $\begin{array}{l}\text { Regulate cell cycle, antiproliferative, } \\
\text { diagnostic and prognostic biomarker, } \\
\text { regulate Tamoxifen response }\end{array}$ & $\begin{array}{l}\text { Bertoli et al. } 2015 \text { [3], Schooneveld et } \\
\text { al. } 2015 \text { [36] } \\
\text { Kaboli et al. } 2015 \text { [58], Leivonen et al. } \\
2014 \text { [91], Romero-Cordoba et al. } 2012 \\
\text { [92] }\end{array}$ \\
\hline 373 & Cell line & Up & CD44 & Cell migration, invasion and metastasis & \\
\hline
\end{tabular}


Table 1 miRNAs expression level, their targets, functional roles and potentials as biomarker in human breast cancer (female) (Continued)

\begin{tabular}{|c|c|c|c|c|c|}
\hline & & & & & $\begin{array}{l}\text { Bertoli et al. } 2015 \text { [3], Schooneveld et } \\
\text { al. } 2015 \text { [36], Shafi et al. } 2014 \text { [32], Fu et } \\
\text { al. } 2011 \text { [62] }\end{array}$ \\
\hline 429 & $\begin{array}{l}\text { Cell line, } \\
\text { Tissue }\end{array}$ & Down & $\begin{array}{l}\text { ZEB1, ZEB2, CRKL, } \\
\text { TUBB2A, TGF- } \beta, \text { XIAP }\end{array}$ & $\begin{array}{l}\text { Anti-proliferative and anti-metastatic, } \\
\text { member of miR-200 family }\end{array}$ & $\begin{array}{l}\text { Takahashi et al. } 2015 \text { [59], Ye et al. } 2015 \\
\text { [93], } \\
\text { Kaboli et al. } 2015 \text { [58], Wang et al. } 2015 \\
\text { [94] }\end{array}$ \\
\hline $491-5 p$ & $\begin{array}{l}\text { Cell line, } \\
\text { Tissue }\end{array}$ & Down & $\begin{array}{l}\text { EGRF, HER2, NNAT, } \\
\text { JMJD2B }\end{array}$ & $\begin{array}{l}\text { Antiproliferative, antimetastatic especially } \\
\text { estrogen stimulated breast cancer cells }\end{array}$ & $\begin{array}{l}\text { Hui et al. } 2015 \text { [95], Kaboli et al. } 2015 \\
\text { [58] }\end{array}$ \\
\hline 495 & $\begin{array}{l}\text { Cell line, } \\
\text { BCSCs }\end{array}$ & Up & REDDI, ECAD & $\begin{array}{l}\text { Increased tumor formation, } \\
\text { downregulation of E-cadherin, maintain- } \\
\text { ing a stem-cell line phenotype }\end{array}$ & $\begin{array}{l}\text { Kaboli et al. } 2015 \text { [58], Schwarzenbacher } \\
\text { et al. } 2013 \text { [61] }\end{array}$ \\
\hline $520 c$ & $\begin{array}{l}\text { Cell line, } \\
\text { Tissue }\end{array}$ & Up & CD44 & $\begin{array}{l}\text { Cell migration, invasion and metastasis, } \\
\text { Tamoxifen response }\end{array}$ & $\begin{array}{l}\text { Bertoli et al. } 2015 \text { [3], Kaboli et al. } 2015 \\
\text { [58] }\end{array}$ \\
\hline 708 & $\begin{array}{l}\text { Tissue, Cell } \\
\text { line }\end{array}$ & Down & NNAT & Anti-proliferative and anti-metastatic & $\begin{array}{l}\text { Kurozumi et al. } 2016 \text { [57], Kaboli et al. } \\
2015 \text { [58], Ryu et al. } 2013 \text { [96] }\end{array}$ \\
\hline
\end{tabular}

cancer, upregulated miRNA210 was reported as a diagnostic and prognostic marker $[3,36]$.

A study reported that protein expression profiles were comparable between canine mammary tumors and human breast cancers. However, 12 other differentially expressed proteins in metastatic canine mammary tumors have not been studied yet and it remains to be established whether they are associated or not with metastasis of human breast cancers [54]. It is noted that researchers are able to study myoepithelial cell proliferation in canine breast cancer model rather than in human breast cancer due to their incidence rate $>20 \%$ and $<0.1 \%$ respectively [55].

Besides the aforementioned similarities between canine and human breast cancers, other aspects that make canine tumors a good model for human breast cancer is faster aging (7 times more) compared to human due to their shorter life span and additionally the average shorter overall survival rate of dogs diagnosed with cancers [56].

\section{Resources of miRNAs database}

MicroRNA database including targets of miRNAs can be retrieved from the following web-based online programs.

1. miRBase: http://www.mirbase.org/

This site was founded by Sam Griffths-Jones and currently governed by Griffiths-Jones lab of University of Manchester, UK. The version 1 of miRNAs database was introduced in 2002 with 218 entries. Total entries became 28, 645 in their latest version 21 that was released in 2014. These entries can be viewed and downloaded by names, keywords, references and annotations of respective miRNAs. This site is also available for naming of newly found miRNAs in order to avoid overlaps.

2. miRwalk: http://zmf.umm.uni-heidelberg.de/apps/ $\mathrm{zmf} /$ mirwalk2/

Current version is miRNA walk 2 and the site handle 15 different species' information on genes, mRNA and miRNA. This website is controlled by University Heidelberg, Faculty of medicine, Germany. Total 11,748 miRNA and 308,700 genes are documents in this version and generate predicted and validated miRNA-target interactions.

\section{3. miRTarBase: http://mirtarbase.mbc.nctu.edu.tw/}

This site was established by Department of Biological Science and Technology, institute of Bioiformatics National Chiao Tung University of Taiwan. This site is mainly used for identifying not only miRNA targets but also to understand interaction networks such as biological functions of miRNAs against their targets in biological pathways (KEGG pathways).

\section{4. miRNAmap: http://mirnamap.mbc.nctu.edu.tw/}

Established from the same organization mentioned above in (3). This site facilitates to search experimental verified microRNAs as well as experimental verified miRNA target genes in human, mouse, rat, and other metazoan genomes. Data recorded in this site includes expression profiles of 224 human miRNAs in 18 major normal tissues.

5. miRGator: http://mirgator.kobic.re.kr/ 
miRGator is based in Seoul, Korea. Current version 3 provides deep sequencing data of miRNAs that facilitate users to comprehend precursor of miRNAs, its sequences, their final products. Furthermore, miRGator has another two features such as miRNA catalogues and Expression profiles, and miRNA-mRNA target relations and expression correlations.

\section{6. miRDB: http://mirdb.org/miRDB/}

This data based was created by the department of radiation oncology, Washington university school of medicine in St. Louis. This web page provides predicted microRNA targets in human, mouse, rat, dog and chicken. This miRDB search engine allows users to search targets of miRNAs by miRNA name directly or gene accession number from GenBank, Gene Symbol and NCBI Gene ID.

\section{PhenomiR: http://mips.helmholtz-muenchen.de/} phenomir/

Established by German Research Center for Environmental Health. Differentially regulated miRNA expression in diseases and other biological processes can be divulged from this site.

8. miRecords: http://c1.accurascience.com/miRecords/

The Predicted Targets component of miRecords generates integrated results from 11 established miRNA target prediction programs such as DIANA-microT, MicroInspector, miRanda, MirTarget2, miTarget, NBmiRTar, PicTar, PITA, RNA22, RNAhybrid, and TargetScan/ TargertScanS.

9. miRGen: http://carolina.imis.athena-innovation.gr/ diana_tools/web/

miRGen, developed at the University of Pennsylvania, generates miRNA gene transcription start sites (TSSs), coupled with genome wide maps of transcription factor (TF) binding sites in order to understand mechanisms of miRNA transcription regulation.

Other useful online miRNA related sites were microrna.org, targetscan.org, ChIPBase, TarBase, starBase, ebi.ac.uk, PmmR (Putative microRNA-microRNA Regulations) and pictar.mdc-berlin.de.

\section{Conclusion}

MicroRNAs can be easily detected with advanced technologies and the continuous investigations supply miRNA as a novel clue for diagnosis and prognosis marker to therapeutic targets. Furthermore, research on delivering miRNAs mimics and/or inhibitors directly into breast cancer tissue to regulate the balance of miRNAs is gaining attention in the field. The miRNA resources mentioned in this paper are useful to generate predicted targeted mRNAs for interested miRNAs candidate. Aberrant miRNA expression in our body such as oncomirs (upregulated miRNAs) and oncosupressors (downregulated miRNAs) could be the sign of diseases and they can be clinically detected by RT-qPCR, digital $\mathrm{PCR}$, microarrays, and next generation sequencing technologies. Signature miRNA profile can be used to distinguish the stages of breast cancer progression and help in early diagnosis, prognosis, and effective treatment for human breast cancer and canine mammary tumor.

\section{Abbreviations}

AGRO2: Protein argonaute-2; DGCR8: Digeorge syndrome chromosomal region 8; KEGG: Kyoto encyclopedia genes and genomes; miR/ miRNA: microRNA; MRI: Magnetic resonance imaging; mRNA: messenger RNA; PAZ: Protein PAZ; PIWI: Protein PIWl; pre-miRNA: precursor microRNA; pri-miRNA: primary microRNA; RISC: RNA-induced silencing complex; TRBP: TAR RNA binding protein; tRNA: transfer RNA

\section{Acknowledgements}

Not applicable.

\section{Funding}

The authors acknowledge the research funding from Trans Disciplinary Research Grant Scheme (TRGS) (5535000) for the financial support.

Availability of data and materials Not applicable.

\section{Authors' contributions}

RMCY wrote and reviewed the manuscripts. CYK constructed and reviewed the manuscript. Both authors read and approved the final manuscript.

\section{Authors' information}

CYK: Associate professor, Department of Biomedical Science, Molecular Biology laboratory, Faculty of medicine and health sciences, University Putra Malaysia. Founder and president of BioMedKL. Technical advisor for molecular diagnostic at Ramsay Sime Darby Medical Centre, Malaysia. RMCY: PhD candidate, Department of Biomedical Science, Molecular Biology laboratory, Faculty of medicine and health sciences, University Putra Malaysia.

Ethics approval and consent to participate

Not applicable.

\section{Consent for publication}

Not applicable.

\section{Competing interests}

The authors declare that they have no competing interests.

\section{Publisher's Note}

Springer Nature remains neutral with regard to jurisdictional claims in published maps and institutional affiliations.

\section{Author details}

'Department of Biomedical Science, Faculty of Medicine and Health Sciences, University Putra Malaysia, 43400 Serdang, Selangor Darul Ehsan, Malaysia. ${ }^{2}$ Centre for Diagnostic Nuclear Imaging, University Putra Malaysia, 43400 Serdang, Selangor Darul Ehsan, Malaysia. ${ }^{3}$ Institute of Bioscience, University Putra Malaysia, 43400 Serdang, Selangor Darul Ehsan, Malaysia. 


\section{Received: 12 June 2017 Accepted: 29 August 2017}

Published online: 26 October 2017

\section{References}

1. Lee R, Feinbaum R, Ambrost V. The C. Elegans Heterochronic gene lin-4 encodes small RNAs with antisense Complementarity to \& II-14. Cell. 1993; 75:843-54.

2. O'Day E, Lal A. MicroRNAs and their target gene networks in breast cancer Breast Cancer Res. 2010;12:201.

3. Bertoli G, Cava C, Castiglioni I. Th e r a n o s t i c s MicroRNAs : new biomarkers for diagnosis, prognosis, therapy prediction and therapeutic tools for breast cancer; 2015. p. 5.

4. Grosshans H, Filipowicz W. Molecular biologyThe expanding world of small RNAs. Nature. 2008;451:414-6. https://doi.org/10.1016/j.devcel.2014.01.009.

5. Lee $\mathrm{Y}$, Kim M, Han JJ, Yeom KH, Lee $\mathrm{S}$, Baek SH, et al. MicroRNA genes are transcribed by RNA polymerase II. EMBO J. 2004;23:4051-60.

6. Filippov V, Solovyev V, Filippova M, Gill SS. A novel type of RNase III family proteins in eukaryotes. Gene. 2000;245:213-21.

7. Gregory RI, Yan K-P, Amuthan G, Chendrimada T, Doratotaj B, Cooch N, et al. The microprocessor complex mediates the genesis of microRNAs. Nature. 2004:432:235-40.

8. Denli AM, Tops BBJ. Plasterk RH a, Ketting RF, Hannon GJ. Processing of primary microRNAs by the microprocessor complex. Nature. 2004:432:231-5.

9. Czech B, Hannon GJ. Small RNA sorting: matchmaking for Argonautes. Nat Rev Genet. 2011;12:19-31. https://doi.org/10.1038/nrg2916.

10. Lund E, Gu S. Nuclear Export of MicroRNA. Science (80- ). 2004;303 January:95-8.

11. Bernstein E. Caudy a a, Hammond SM, Hannon GJ. Role for a bidentate ribonuclease in the initiation step of RNA interference. Nature. 2001:409:363-6.

12. Chendrimada TP, Gregory RI, Kumaraswamy E, Cooch N, Nishikura K, Shiekhattar R. NIH Public Access. 2010;436:740-4.

13. Lau P-W, Guiley KZ, De N, Potter CS, Carragher B, MacRae IJ. The molecular architecture of human dicer. Nat Struct Mol Biol. 2012;19:436-40. https://doi. org/10.1038/nsmb.2268.

14. Bartel DP. MicroRNAs: genomics, biogenesis, mechanism, and function. Cell. 2004;116:281-97.

15. Ghildiyal M, Xu J, Seitz H, Weng Z, Zamore PD. Sorting of drosophila small silencing RNAs partitions microRNA* strands into the RNA interference pathway. RNA. 2010;16:43-56.

16. Cheng G. Circulating miRNAs: roles in cancer diagnosis, prognosis and therapy. Adv Drug Deliv Rev. 2015;81:75-93. https://doi.org/10.1016/j.addr. 2014.09.001.

17. Okamura K, Hagen JW, Duan H, Tyler DM, Lai EC. NIH Public Access. 2009; 130:89-100

18. Cheloufi S, Dos Santos CO, Chong M. Hannon G. Ago Catalysis. 2010;465:584-9.

19. Yang J-S, Maurin T, Robine N, Rasmussen KD, Jeffrey KL, Chandwani R, et al. Conserved vertebrate mir-451 provides a platform for dicer-independent, Ago2 mediated microRNA biogenesis. Proc Natl Acad Sci U S A. 2010;107:15163-8.

20. Chou C-H, Chang N-W, Shrestha S, Hsu S-D, Lin Y-L, Lee W-H, et al. miRTarBase 2016: updates to the experimentally validated miRNA-target interactions database. Nucleic Acids Res. 2015;44 November 2015:gkv1258-. doi:https://doi.org/10.1093/nar/gkv1258.

21. He L, Hannon GJ. MicroRNAs: small RNAs with a big role in gene regulation. Nat Rev Genet. 2004;5:522-31.

22. Dalmay T. Mechanism of miRNA-mediated repression of mRNA translation Essays Biochem. 2013;54:29-38.

23. Saxena S, Jónsson ZO, Dutta A. Small RNAs with imperfect match to endogenous mRNA repress translation. Implications for off-target activity of small inhibitory RNA in mammalian cells. J Biol Chem. 2003;278:44312-9.

24. MacFarlane L-A, Murphy PR. MicroRNA: biogenesis, function and role in cancer. Curr Genomics. 2010;11:537-61. https://doi.org/10.2174/ 138920210793175895

25. Eiring AM, Harb JG, Neviani P, Garton C, Oaks JJ, Spizzo R, et al. miR-328 functions as an RNA decoy to modulate hnRNP E2 regulation of mRNA translation in leukemic blasts. Cell. 2010;140:652-65. https://doi.org/10.1016/ j.cell.2010.01.007.

26. Tang J, Ahmad A, Sarkar FH. The role of MicroRNAs in breast cancer migration, invasion and metastasis. Int J Mol Sci. 2012;13:13414-37. https:// doi.org/10.3390/ijms131013414

27. Baffa R, Fassan M, Volinia S, O'Hara B. MicroRNA expression profiling of human metastatic cancers identifies cancer gene targetst. J Pathol. 2009;219
28. Henry JC, Azevedo-Pouly ACP, Schmittgen TD. MicroRNA replacement therapy for cancer. Pharm Res. 2011;28:3030-42.

29. Christodoulatos GS, Dalamaga M. Micro-RNAs as clinical biomarkers and therapeutic targets in breast cancer: quo vadis? World J Clin Oncol. 2014;5: 71-81. https://doi.org/10.5306/wjco.v5.i2.71.

30. Piva R. Spandidos D a., Gambari R. From microRNA functions to microRNA therapeutics: novel targets and novel drugs in breast cancer research and treatment (review). Int J Oncol. 2013;43:985-94.

31. Blenkiron C, Goldstein LD, Thorne NP, Spiteri I, Chin S-F, Dunning MJ, et al. MicroRNA expression profiling of human breast cancer identifies new markers of tumor subtype. Genome Biol. 2007;8:R214.

32. Shafi G, Hasan TN, Syed NA, Paine A, Tegner J, Munshi A. Omics approaches in breast cancer; 2014. p. 171-82. https://doi.org/10.1007/978-81-322-0843-3.

33. Hurst DR, Edmonds MD, Welch DR. Metastamir: the field of metastasisregulatory microRNA is spreading. Cancer Res. 2009:69:7495-8.

34. Lopez-Camarillo C, Marchat LA, Arechaga-Ocampo E, Perez-Plasencia C, del OralHernandez O, Castaneda-Ortiz EJ, et al. MetastamiRs: non-coding microRNAs driving cancer invasion and metastasis. Int J Mol Sci. 2012;13:1347-79.

35. Ma L, Reinhardt F, Pan E, Soutschek J, Bhat B, Teruya-feldstein J, et al. Therapeutic silencing of miR-10b inhibits metastasis in a mouse mammary tumor model. Nat Biotechnol. 2010;28:341-7.

36. Schooneveld E, Wildiers H, Vergote I, Vermeulen PB, Dirix LY, Van Laere SJ. Dysregulation of microRNAs in breast cancer and their potential role as prognostic and predictive biomarkers in patient management. Breast Cancer Res. 2015;17:1-15. https://doi.org/10.1186/s13058-015-0526-y.

37. Várallyay E, Burgyán J, Havelda Z. MicroRNA detection by northern blotting using locked nucleic acid probes. Nat Protoc. 2008:3:190-6.

38. Wang Z, Yang B. MicroRNA expression detection methods. 1st Editio. Springer-Verlag: Berlin Heidelberg; 2010. doi:https://doi.org/10.1007/ 9783642049286

39. Balcells I, Cirera S, Busk PK. Specific and sensitive quantitative RT-PCR of miRNAs with DNA primers. BMC Biotechnol. 2011;11:70. https://doi.org/10. 1186/1472-6750-11-70

40. Creighton CJ, Reid JG, Gunaratne PH. Expression profiling of microRNAs by deep sequencing. Brief Bioinform. 2009;10:490-7.

41. Mattiske S, Suetani RJ, Neilsen PM, Callen DF. The oncogenic role of miR155 in breast cancer. Cancer Epidemiol Biomark Prev. 2012;21:1236-43.

42. Han J-G, Jiang Y-D, Zhang C-H, Yang Y-M, Pang D, Song Y-N, et al. A novel panel of serum miR-21/miR-155/miR-365 as a potential diagnostic biomarker for breast cancer. Ann Surg Treat Res. 2017;92:55-66. https://doi. org/10.4174/astr.2017.92.2.55.

43. Queiroga FL, Raposo T, Carvalho MI, Prada J, Pires I. Canine mammary tumours as a model to study human breast cancer: most recent findings. In Vivo. 2011;25:455-65.

44. Strandberg JD, Goodman DG. Animal model of human disease: canine mammary neoplasia. Am J Pathol. 1974;75:225-8.

45. Schneider R. Comparison of age, sex, and incidence rates in human and canine breast cancer. Cancer. 1970;26:419-26.

46. Prier JE, Brodey RS. Canine Neoplasia: a prototype for human cancer study; 1959. p. 331-44.

47. Sorenmo KU. Canine mammary gland tumors. Vet Clin North Am Small Anim Pract. 2003;33:573-96. https://doi.org/10.1016/S0195-5616(03)00020-2.

48. Loukopoulos P, Mungall B a, Straw RC, Thornton JR, Robinson WF. Matrix metalloproteinase-2 and -9 involvement in canine tumors. Vet Pathol. 2003;40:382-94

49. Shah FD, Shukla SN, Shah PM, Shukla HK, Patel PS. Clinical significance of matrix metalloproteinase 2 and 9 in breast cancer. Indian J Cancer. 2013;46: 194-202. https://doi.org/10.4103/0019-509X.52953.

50. Wagner S, Willenbrock S, Nolte I, Murua EH. Comparison of non-coding RNAs in human and canine cancer. Front Genet. 2013:46. https://doi.org/10. 3389/fgene.2013.00046.

51. Boggs RM, Wright ZM, Stickney MJ, Porter WW, Murphy KE. MicroRNA expression in canine mammary cancer. Mamm Genome. 2008;19:561-9.

52. Zhou D, Li S, Wen J, Gong X, Xu L, Luo Y. Genome-wide computational analyses of microRNAs and their targets from Canis Familiaris. Comput Biol Chem. 2008;32:60-5

53. von Deetzen M-C, Schmeck BT, Gruber AD, Klopfleisch R. Malignancy associated MicroRNA expression changes in canine mammary cancer of different malignancies. ISRN Vet Sci. 2014;2014:148597. https:/doi.org/10.1155/2014/148597.

54. Klopfleisch R, Klose P, Weise C, Bondzio A, Multhaup G, Einspanier R, et al. Proteome of metastatic canine mammary carcinomas : similarities to and differences from human breast cancer + research articles; 2010. p. 6380-91. 
55. Liu D, Xiong H, Ellis AE, Northrup NC, Rodriguez CO Jr, O'Regan RM, et al. Molecular homology and difference between spontaneous canine mammary cancer and human breast cancer. Cancer Res. 2014;74:5045-56.

56. Hawai SM, Ali MM, Niu Y, Alawad A, Aljofan M. Dogs : active role model for cancer studies - a review. J Cancer Ther. 2013;2013 July:989-95.

57. Kurozumi S, Yamaguchi Y, Kurosumi M, Ohira M, Matsumoto H, Horiguchi J. Recent trends in microRNA research into breast cancer with particular focus on the associations between microRNAs and intrinsic subtypes. J Hum Genet. 2016;62:15-24. https://doi.org/10.1038/jhg.2016.89.

58. Kaboli PJ, Rahmat A, Ismail P, Ling K-H. MicroRNA-based therapy and breast cancer: a comprehensive review of novel therapeutic strategies from diagnosis to treatment. Pharmacol Res. 2015;97:104-21. https://doi.org/10. 1016/j.phrs.2015.04.015.

59. Takahashi RU, Miyazaki H, Ochiya T. The roles of microRNAs in breast cancer. Cancers (Basel). 2015;7:598-616.

60. Zhang W-C, Liu J, Xu X, Wang G. The role of microRNAs in breast cancer progression. Med Oncol. 2014;30:675. https://doi.org/10.1007/s12032-013-0675-8.

61. Schwarzenbacher D, Balic M, Pichler M. The role of microRNAs in breast cancer stem cells. Int J Mol Sci. 2013;14:14712-23.

62. Fu SW, Chen L, Man Y-G. miRNA biomarkers in breast cancer detection and management. J Cancer. 2011;2:116-22.

63. Xiang J, Wu J. Feud or friend? The role of the miR-17-92 cluster in tumorigenesis. Curr Genomics. 2010;11:129-35. https://doi.org/10.2174/ 138920210790886853.

64. Bonauer A, Dimmeler S. The microRNA-17 92 cluster: still a miRacle? Cell Cycle. 2009;:8:3866-73.

65. Kodahl AR, Lyng MB, Binder H, Cold S, Gravgaard K, Knoop AS, et al. Novel circulating microRNA signature as a potential non-invasive multi-marker test in ER-positive early-stage breast cancer: a case control study. Mol Oncol. 2014;8:874-83. https://doi.org/10.1016/j.molonc.2014.03.002.

66. Tang W, Zhu J, Su S, Wu W, Liu Q, Su F, et al. MiR-27 as a prognostic marker for breast cancer progression and patient survival. PLoS One. 2012;7:e51702. https://doi.org/10.1371/journal.pone.0051702.

67. Mertens-Talcott SU, Chintharlapalli S, Li X, Safe S. The oncogenic microRNA-27a targets genes that regulate specificity protein transcription factors and the G2-M checkpoint in MDA-MB-231 breast cancer cells. Cancer Res. 2007;67:11001-11.

68. Ding L, Ni J, Yang F, Huang L, Deng H, Wu Y, et al. Promising therapeutic role of miR-27b in tumor. Tumor Biol. 2017;39:101042831769165. https://doi. org/10.1177/1010428317691657.

69. Hsieh T-H, Hsu C-Y, Tsai C-F, Long C-Y, Wu C-H, Wu D-C, et al. HDAC inhibitors target HDAC5, Upregulate MicroRNA-125a-5p, and induce apoptosis in breast cancer cells. Mol Ther. 2015;23:656-66. https://doi.org/ 10.1038/mt.2014.247.

70. Feliciano A, Castellvi J, Artero-Castro A, Leal JA, Romagosa C, HernándezLosa J, et al. miR-125b acts as a tumor suppressor in breast tumorigenesis via its novel direct targets ENPEP, CK2-a, CCNJ, and MEGF9. PLoS One. 2013; 8:1-18.

71. Wang H, Tan G, Dong L, Cheng L, Li K, Wang Z, et al. Circulating mir-125b as a marker predicting chemoresistance in breast cancer. PLoS One. 2012;7: e34210. https://doi.org/10.1371/journal.pone.0034210.

72. Zheng Y, Lv X, Wang X, Wang B, Shao X, Huang Y. miR-181b promotes chemoresistance in breast cancer by regulating Bim expression; 2016. p. 683-90.

73. Calura E, Martini P, Sales G, Beltrame L, Chiorino G, D'Incalci M, et al. Wiring miRNAs to pathways: a topological approach to integrate miRNA and mRNA expression profiles. Nucleic Acids Res. 2014;42:e96. https://doi.org/10. 1093/nar/gku354.

74. Le XF, Almeida MI, Mao W, Spizzo R, Rossi S, Nicoloso MS, et al. Modulation of microrna-194 and cell migration by her2-targeting trastuzumab in breast cancer. PLoS One. 2012;7:1-14.

75. Fang C, Wang F-B, Li Y, Zeng X-T. Down-regulation of miR-199b-5p is correlated with poor prognosis for breast cancer patients. Biomed Pharmacother. 2016;84:1189-93. https://doi.org/10.1016/j.biopha.2016.10.006.

76. Fang C, Zhao Y, Guo B. MiR-199b-5p targets HER2 in breast cancer cells. J Cell Biochem. 2013;114:1457-63.

77. Hilmarsdottir B, Briem E, Bergthorsson J, Magnusson M, Gudjonsson T. Functional role of the microRNA-200 family in breast morphogenesis and Neoplasia. Genes (Basel). 2014;5:804-20. https://doi.org/10.3390/ genes5030804.

78. Tsouko E, Wang J, Frigo DE, Aydogdu E, Williams C. miR-200a inhibits migration of triple-negative breast cancer cells through direct repression of the EPHA2 oncogene. Carcinogenesis. 2015;36:1051-60. https://doi.org/10. 1093/carcin/bgv087.

79. Yao Y, Hu J, Shen Z, Yao R, Liu S, Li Y, et al. MiR-200b expression in breast cancer: a prognostic marker and act on cell proliferation and apoptosis by targeting Sp1. J Cell Mol Med. 2015;19:760-9.

80. Ye F, Tang H, Liu Q, Xie X, Wu M, Liu X, et al. MiR-200b as a prognostic factor targets multiple members of RAB family in glioma. Med Oncol. 2014; 31:1-10.

81. Jurmeister S, Baumann M, Balwierz A, Keklikoglou I, Ward A, Uhlmann S, et al. MicroRNA-200c represses migration and invasion of breast cancer cells by targeting Actin-regulatory proteins FHOD1 and PPM1F. Mol Cell Biol. 2012;32:633-51.

82. Shimono Y, Ugalde M, Cho R, Lobo N, Dalerba P, Qian D, et al. Downregulation of miRNA-200c links breast cancer stem cells with normal stem cells. Cell. 2009:138:423-4.

83. Shen S-Q, Huang L-S, Xiao X-L, Zhu X-F, Xiong D-D, Cao X-M, et al. miR-204 regulates the biological behavior of breast cancer MCF-7 cells by directly targeting FOXA1. Oncol Rep. 2017:1-9. https://doi.org/10.3892/or.2017.5644.

84. Li T, Pan H, Li R. The dual regulatory role of miR-204 in cancer. Tumor Biol. 2016;37:11667-77. https://doi.org/10.1007/s13277-016-5144-5.

85. Flores-Pérez A, Marchat LA, Rodríguez-Cuevas S, Bautista-Piña V, HidalgoMiranda A, Ocampo EA, et al. Dual targeting of ANGPT1 and TGFBR2 genes by miR-204 controls angiogenesis in breast cancer. Sci Rep. 2016;6:34504. https://doi.org/10.1038/srep34504.

86. Devlin C, Greco S, Martelli F, Ivan M. MiR-210: more than a silent player in hypoxia. IUBMB Life. 2011;63:94-100.

87. Huang X, Ding L, Bennewith K, Tong R, Ang KK, Le Q, et al. Hypoxia inducible mir-210 regulates normoxic gene expression involved in tumor initiation. 2010;35:856-867.

88. Jana S, Sengupta S, Biswas S, Chatterjee A, Roy H, Bhattacharyya A. miR216b suppresses breast cancer growth and metastasis by targeting SDCBP. Biochem Biophys Res Commun. 2017;482:126-33. https://doi.org/10.1016/j. bbrc.2016.10.003.

89. Zhao L, Ren Y, Tang H, Wang W, He Q, Sun J, et al. Deregulation of the miR222-ABCG2 regulatory module in tongue squamous cell carcinoma contributes to chemoresistance and enhanced migratory/invasive potential. Oncotarget. 2015;6:44538-50.

90. Liu F, Liu Y, Shen J, Zhang G, Han J. MicroRNA-224 inhibits proliferation and migration of breast cancer cells by down-regulating fizzled 5 expression. Oncotarget. 2014; 10.18632/oncotarget.9734.

91. Leivonen SK, Sahlberg KK, Mäkelä R, Due EU, Kallioniemi O, Børresen-Dale AL, et al. High-throughput screens identify microRNAs essential for HER2 positive breast cancer cell growth. Mol Oncol. 2014;8:93-104.

92. Romero-Cordoba S, Rodriquez-Cuevas S, Rebollar-Vega R, Quintanar-Jurado $\checkmark$, Maffuz-Aziz A, Jimenez-Sanchez G, et al. Identification and pathway analysis of microRNAs with no previous involvement in breast cancer. PLoS One. 2012;7

93. Ye Z-B, Ma G, Zhao Y-H, Xiao Y, Zhan Y, Jing C, et al. miR-429 inhibits migration and invasion of breast cancer cells in vitro. Int J Oncol. 2015;46:531-8.

94. Wang C, Ju H, Shen C, Tong Z. miR-429 mediates $\delta$-tocotrienol-induced apoptosis in triple-negative breast cancer cells by targeting XIAP. Int J Clin Exp Med. 2015;8:15648-56. https://doi.org/10.1093/carcin/bgv087.

95. Hui Z, Yiling $C$, Wenting $Y$, Xuqun H, Chuanyi Z, Hui L. miR-491-5p functions as a tumor suppressor by targeting JMJD2B in ER a -positive breast cancer. FEBS Lett. 2015;589:812-21. https://doi.org/10.1016/j.febslet.2015.02.014.

96. Ryu S, McDonnell K, Choi H, Gao D, Hahn M, Joshi N, et al. Suppression of miRNA-708 by Polycomb group promotes metastases by calcium-induced cell migration. Cancer Cell. 2013;23:63-76. https:/doi.org/10.1016/j.ccr.2012.11.019. 